\title{
Application of Double Fourier Series for Analyzing Harmonics of a Modular Multilevel Converter
}

\author{
Ngoc-Thinh Quach ${ }^{1}$, Sang Heon Chae ${ }^{1}$, Min-Hyeok Kang ${ }^{1}$, Dong Wan Kim², \\ Seung Hun $\mathrm{Ko}^{2}$ and Eel-Hwan Kim ${ }^{1}$ \\ ${ }^{1}$ Department of Electrical Engineering \\ Jeju National University \\ Jeju-si, Jeju Special Self-Governing Province, 63243, Republic of Korea \\ Phone/Fax number: +82 64 7543678, e-mail: ngocthinh@ctu.edu.vn, chae@jejunu.ac.kr, minh0131@jejunu.ac.kr, \\ ehkim@jejunu.ac.kr \\ ${ }^{2}$ Research \& Development Center, Jeju Energy Corporation \\ Jeju-si, Jeju Special Self-Governing Province, 63243, Republic of Korea \\ E-mail: segi305@jejuenergy.or.kr, kosh5862@jejuenergy.or.kr
}

\begin{abstract}
This paper presents an application of double Fourier series (DFS) algorithm for analyzing harmonics of a modular multilevel converter (MMC). By considering the effect of arm inductor, the application of DFS for calculating harmonics in the MMC is made. The DFS is then verified by comparing with simulation results used the fast Fourier transform (FFT) algorithm. Subsequently, the DFS will be used to analyze the effect of number of output voltage levels to the distribution of harmonics and total harmonic distortion (THD) in the MMC. The analytical results are performed in the Matlab programming in order to verify the theoretical analysis.
\end{abstract}

Key words: Double Fourier series, Fast Fourier transform, Harmonic analysis, Modular multilevel converter, Total harmonic distortion

\section{Introduction}

The modular multilevel converter (MMC) has been developed for medium and high voltage applications in recent years. The operation principles of MMC have been studied by many authors over the world [1, 2]. Although in low total harmonic distortion (THD), the MMC also injects the harmonics into AC side of the power system. Moreover, the distribution of harmonics changes according to the operating condition. An analysis of harmonics is quite complex and it is often done by using fast Fourier transform (FFT) algorithm. This method offers the benefits of expediency and reduces mathematical effort, but requires considerable computing capacity at a higher carrier frequency. Besides, the FFT often cause a slight error to the obtained results because this method is quite sensitive to both the time resolution of simulation and the periodicity of the overall waveform. In contrast, an analytical method which can calculate the harmonics with a higher precision has been introduced by
Bennett [3] for communication applications. This method is named by a double Fourier series (DFS) algorithm. During the last years, the application of DFS for analyzing harmonics of the voltage source converter (VSC) topologies, such as single-phase VSC, three-phase VSC, diode-clamped and cascaded multilevel converters, has been researched by many authors [4]-[7]. However, there is no any research on application of DFS for the MMC.

Therefore, this paper presents an application of DFS algorithm for analyzing harmonics of the MMC. The objective is to determine the distribution of harmonics as well as the THD in the MMC. To do this, the application of DFS for calculating harmonics in the MMC is first made by considering the effect of arm inductor. The DFS is verified by comparing with simulation results used the FFT. Then, the effect of number of output voltage levels to the distribution of harmonics as well as the THD in the MMC will be analyzed.

\section{Modular Multilevel Converter}

The basic structure of the MMC is shown in Fig. 1. If there are $N$ submodules (SMs) per each arm, the number of levels in output voltage will be $N+1$. The MMC with $N+1$ levels in output voltage is called by $N+1-$ level MMC. To turn on and off for the IGBTs in each SM, the pulse width modulation (PWM) methods are used. One of them is the sinusoidal PWM (SPWM) method that a sinusoidal reference signal is compared with carrier waveforms to generate the gating signals for the IGBTs. The MMC with $N$ SMs per arm requires $N$ carrier waveforms.

In this analysis, the phase-shifted PWM (PS-PWM) method will be applied for the MMC because this is a simple modulation method. In the PS-PWM method, $N$ carrier waveforms have the same frequency and the peak- 


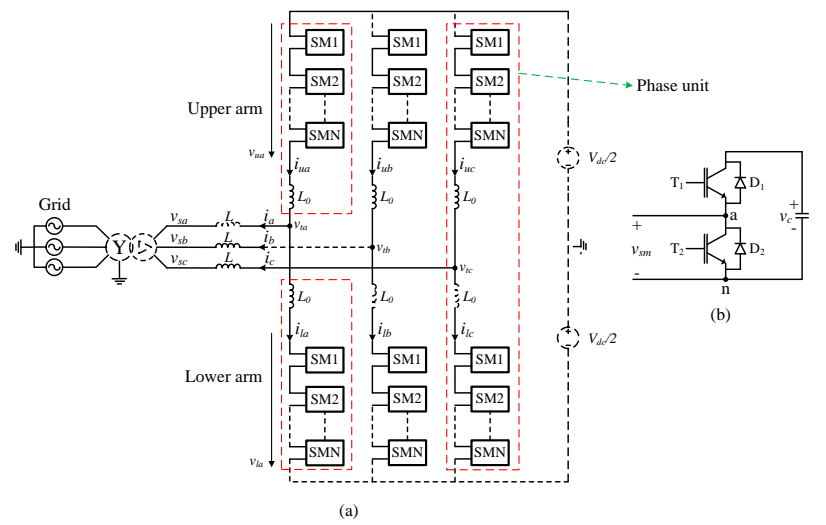

Fig. 1. Configuration of the MMC.

(a) Circuit diagram, (b) Submodule

to-peak amplitude, but there is a phase shift between two adjacent carrier waveforms.

The arrangement of the carrier waveforms can be explained by the phase shift between the first carrier waveform and $N$ th carrier waveform as follows.

$$
\varphi_{i}=\frac{2 \pi(i-1)}{N}(\mathrm{rad}) \quad i=1,2,3, \ldots, N
$$

The total switching frequency, $f_{c t}$, of $N$ SMs in each arm will be equal to $N . f_{c}\left(f_{c t}=N\right.$. $\left.f_{c}\right)$.

\section{Analyzing harmonics with double Fourier series}

From the DFS theory, any periodic function $f(t)$ in the 3-D space $x y z$ can be expressed as

$$
\begin{aligned}
z=f(t)=f(x, y)= & \frac{A_{00}}{2}+\sum_{n=1}^{\infty}\left[A_{0 n} \cos n y+B_{0 n} \sin n y\right] \\
& +\sum_{m=1}^{\infty}\left[A_{m 0} \cos m y+B_{m 0} \sin m y\right] \\
& +\sum_{\substack{m=1 \\
m}}^{\infty} \sum_{\substack{n=-\infty \\
n \neq 0)}}^{\infty}\left[A_{m n} \cos (m x+n y)+B_{m n} \sin (m x+n y)\right]
\end{aligned}
$$

where

$$
\begin{aligned}
\bar{C}_{m n} & =A_{m n}+j B_{m n}=\frac{1}{2 \pi^{2}} \int_{-\pi}^{\pi} \int_{-\pi}^{\pi} f(x, y) e^{j(m x+n y)} d x d y \\
y & =\omega_{0} t+\theta_{0} \\
x & =\omega_{c} t+\theta_{c}
\end{aligned}
$$

$\omega_{0}$ and $\omega_{c}$ are angular frequencies of the fundamental output waveform and the carrier waveform; $\theta_{0}$ and $\theta_{c}$ are initial phase angles of the fundamental output waveform and the carrier waveform, and they are set to zero in this paper $\left(\theta_{0}=\theta_{c}=0\right)$.

In this paper, the DFS is used to analyze the harmonics in a PWM voltage source converter. Fig. 2 illustrates the generation of output voltage of a single-phase PWM converter (Submodule in Fig. 1(b)) with the DFS and the conventional PWM methods. It is demonstrated that these two methods are equivalent in generating the PWM output voltage waveform.

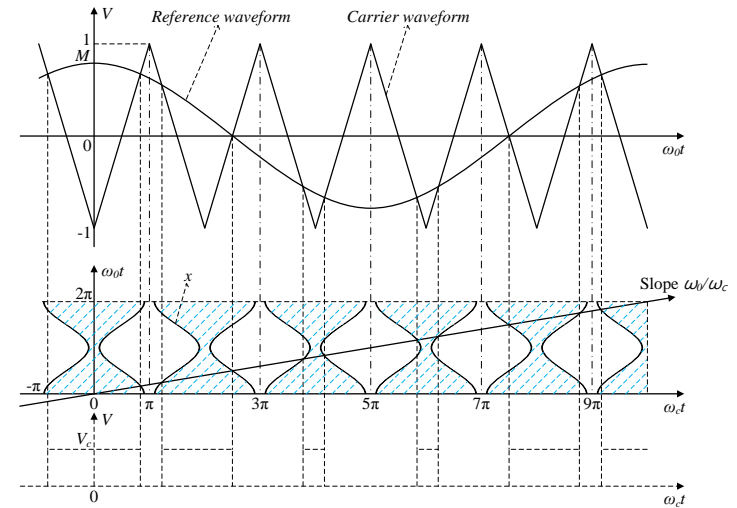

Fig. 2. Conventional PWM method and 2-D model of DFS

Solving (3) and substituting into (2), the harmonic solution for the output voltage of the SM, $v_{s m}(t)$, can be achieved as follows.

$$
\begin{aligned}
& v_{s m}(t)=\frac{V_{c}}{2}+\frac{V_{c}}{2} M \cos \omega_{0} t \\
& +\frac{2 V_{c}}{\pi} \sum_{m=1}^{\infty} \frac{1}{m} J_{0}\left(m \frac{\pi}{2} M\right) \sin \left(m \frac{\pi}{2}\right) \cos \left(m \omega_{c} t\right) \\
& +\frac{2 V_{c}}{\pi} \sum_{m=1}^{\infty} \sum_{\substack{n=-\infty \\
n \neq 0}}^{\infty} \frac{1}{m} J_{n}\left(m \frac{\pi}{2} M\right) \sin \left([m+n] \frac{\pi}{2}\right) \cos \left(m \omega_{c} t+n \omega_{0} t\right)
\end{aligned}
$$

where $J_{0}($.$) and J_{n}($.$) are the Bessel functions. V_{c}$ is the capacitor voltage of the SM, and it is determined by

$$
V_{c}=\frac{V_{d c}}{N}
$$

Eq. (6) shows the solution for the output voltage of the SM. If the voltage drop on arm inductor is ignored, the lower arm voltage of the phase A will be a sum of the output voltages of $N$ SMs per arm. For all $m \neq k N(k=1$, $2, \ldots, \infty)$, the output voltage of phase A, $v_{a}(t)$, can be achieved as

$$
\begin{aligned}
v_{a}(t)= & \frac{N V_{c}}{2} M \cos \omega_{0} t+\frac{2 V_{c}}{\pi} \sum_{m=1}^{\infty} \frac{1}{m} J_{0}\left(N m \frac{\pi}{2} M\right) \sin \left(N m \frac{\pi}{2}\right) \cos \left(N m \omega_{c} t\right) \\
& +\frac{2 V_{c}}{\pi} \sum_{\substack{m=1 \\
\infty}}^{\infty} \sum_{\substack{n=-\infty \\
n \neq 0}}^{\infty} \frac{1}{m} J_{n}\left(N m \frac{\pi}{2} M\right) \sin \left([N m+n] \frac{\pi}{2}\right) \cos \left(N m \omega_{c} t+n \omega_{0} t\right)
\end{aligned}
$$

Then, the line-to-line output voltage between phase A and phase $\mathrm{B}, v_{a b}(t)$, of the MMC is calculated as

$$
\begin{aligned}
v_{a b}(t)= & \frac{\sqrt{3} N V_{c}}{2} M \cos \left(\omega_{0} t+\frac{\pi}{6}\right)+ \\
& +\frac{4 V_{c}}{\pi} \sum_{\substack { m=1 \\
\begin{subarray}{c}{n=-\infty \\
n \neq 0{ m = 1 \\
\begin{subarray} { c } { n = - \infty \\
n \neq 0 } }\end{subarray}}^{\infty} \frac{1}{m} J_{n}\left(N m \frac{\pi}{2} M\right) \sin \left([N m+n] \frac{\pi}{2}\right) . \\
& . \sin \left(\frac{n \pi}{3}\right) \cos \left(N m \omega_{c} t+n \omega_{0} t-\frac{n \pi}{3}+\frac{\pi}{2}\right)
\end{aligned}
$$

There are some notices for the line-to-line output voltage in (9) as follows.

+ There are no baseband harmonics.

+ The carrier harmonics don't appear because they are the same for all phases.

+ The sideband harmonics with even combinations of $\mathrm{Nm}+n$ and triplen sideband harmonics will be zero. 
+ The sideband hamonics center around the frequency of $k N f_{c}(k=1,2, \ldots, \infty)$.

Eq. (9) expresses for an ideal line-to-line output voltage without considering the voltage drop on arm inductor. However, there is still a voltage drop on arm inductor in practice. Thus, the effect of arm inductor should be taken in account. From Fig. 1, if the AC grid is replaced by a series RL load $\left(Z=R_{1}+\mathrm{j} \omega L_{1}\right)$, the ideal phase output voltage can be expressed as

$$
v_{j}=\frac{v_{l j}-v_{u j}}{2}=\frac{L_{0}}{2} \frac{d i_{j}}{d t}+i_{j}\left(R_{1}+j \omega_{0}\left(L+L_{1}\right)\right)
$$

where $j=a, b, c$ denotes phase $\mathrm{A}, \mathrm{B}$, and $\mathrm{C}$.

Assuming that PWM converter generates the harmonics into the output voltage and current. At a steady-state, the harmonic currents can be calculated as

$$
i_{j h}=\frac{v_{j h}}{Z_{1}}=\frac{v_{j h}}{R_{1}+j \omega_{h}\left(\frac{L_{0}}{2}+L+L_{1}\right)}
$$

where $h$ is the harmonic order, $h=2,3, \ldots, \infty$ and $\omega_{h}=2 \pi f_{0} h$.

Thus, the harmonic components of real phase voltage and real line-to-line voltage will be expressed as

$$
\begin{aligned}
& v_{t j h}=v_{j h}-i_{j h}\left(j \frac{\omega_{h} L_{0}}{2}\right) \\
& v_{t a b h}=v_{t a h}-v_{t b h}
\end{aligned}
$$

where $v_{t j}$ is the real phase output voltage of phase $j$ that is considering the effect of arm inductor.

\section{Simulation results}

In this Section, the simulated results and the analytical results are obtained by using PSCAD/EMTDC simulation program and Matlab programming, respectively. The parameters of the MMC are given in Table 1.

Table 1. The parameters of MMC

\begin{tabular}{|l|c|}
\hline \multicolumn{1}{|c|}{ Quantity } & Value \\
\hline Active power $(P)$ & $20 \mathrm{MW}$ \\
\hline Reactive power $(Q)$ & $15 \mathrm{MVar}$ \\
\hline AC voltage $\left(V_{s}\right)$ & $22.9 \mathrm{kV}$ \\
\hline Fundamental frequency $\left(f_{0}\right)$ & $60 \mathrm{~Hz}$ \\
\hline Carrier frequency $\left(f_{c}\right)$ & According to analysis \\
\hline Transformer ratio & $22.9 \mathrm{kV} / 12 \mathrm{kV}$ \\
\hline DC-link voltage $\left(V_{d c}\right)$ & $24 \mathrm{kV}$ \\
\hline Number of SMs per arm $(N)$ & According to analysis \\
\hline Modulation index $(M)$ & According to analysis \\
\hline
\end{tabular}

\subsection{Comparison between DFS algorithm and FFT algorithm}

This section is to verify the DFS algorithm in Section 3. The carrier frequency, number of SMs per arm and modulation index are set to $360 \mathrm{~Hz}, 10$ and 0.8165 , respectively.

Fig. 3 shows a comparison between the analytical results used DFS and the simulated results used FFT. As seen in Figs. 3(a) and (b), two algorithms can bring to the same results. The THDs obtained by using DFS and FFT are about $8.23 \%$ and $8.07 \%$, respectively. The THD error between two algorithms is not significant. Therefore, the precision of the DFS algorithm is confirmed. The total switching frequency is $3600 \mathrm{~Hz}\left(f_{c t}=3600 \mathrm{~Hz}\right)$ that is corresponding to $60^{\text {th }}$ harmonic order. In the line-to-line voltage, there are no baseband harmonics, carrier harmonics, the sideband harmonics with even combinations of $10 . m+n$ and triplen sideband harmonics of $h .3^{\text {th }}(h=1,2, \ldots, \infty)$. The sideband harmonics are centered around the harmonic order of $k .60^{\text {th }}(k=1,2, \ldots$, $\infty)$. These results exactly match the theoretical analysis in Section 3.

The dependence of the FFT on the simulation time step is expressed in Figs. 3(b) and (c). With the simulation

time step of $10 \mu \mathrm{s}$, the simulated results showed in Fig. 3(c) contain some harmonics (smaller than $10^{-3}$ ) that do not exist in the analytical results or in the simulated results obtained by using the simulation time step of 0.5 $\mu$ s as shown in Fig. 3(b). However, the executive time of simulated results in Fig. 3(b) is extremely long. This shows out the advantage of the DFS in comparing with the FFT.

\subsection{Relationship between harmonics and number of levels}

The number of SMs per arm will be changed in order to analyze the distribution of harmonics versus number of levels of MMC. The analytical results are shown in Fig. 4. The modulation index is the same with Section 4.1. The carrier frequencies are $900 \mathrm{~Hz}, 360 \mathrm{~Hz}$ and $180 \mathrm{~Hz}$ corresponding to 5-level, 11-level and 21-level MMCs, respectively. These carrier frequencies ensure that the

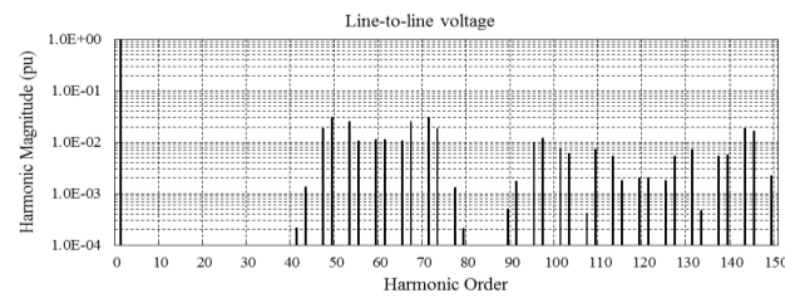

(a)

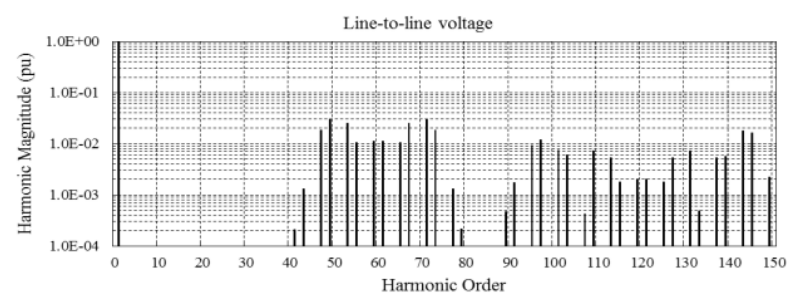

(b)

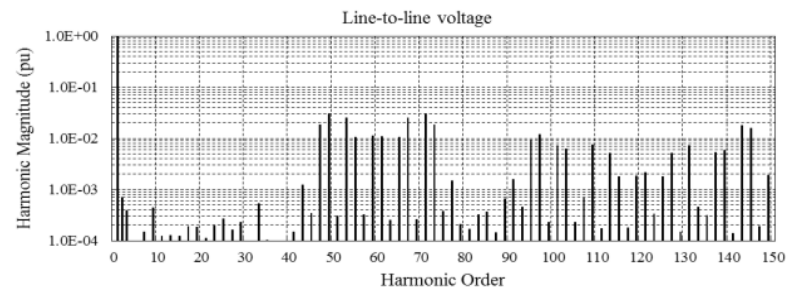

(c)

Fig. 3. Analytical results and simulated results. (a) Analytical results, (b) Simulated results with simulation time step of $0.5 \mu \mathrm{s}$, (c) Simulated results with simulation time step of $10 \mu \mathrm{s}$ 


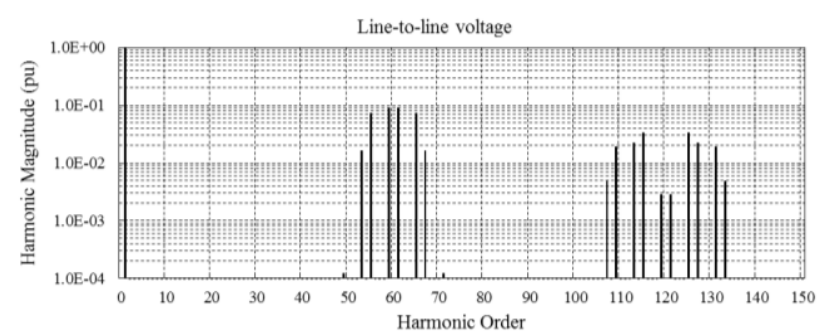

(a)

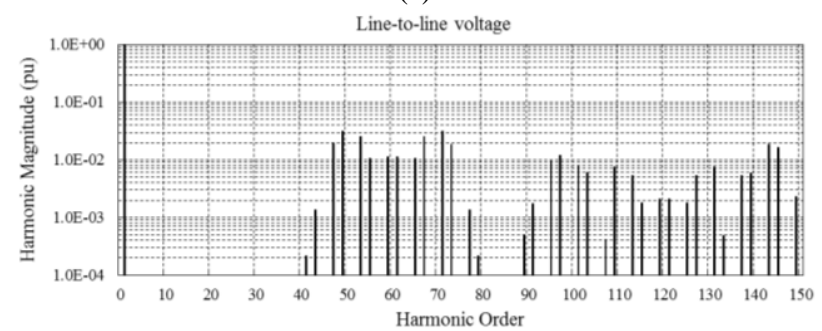

(b)

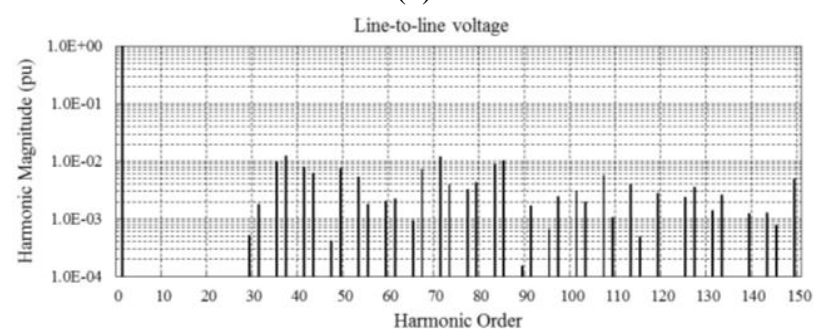

(c)

Fig. 4. Distribution of harmonics versus number of levels of MMC ( $f_{c t}=3600 \mathrm{~Hz}$ ). (a) 5-level MMC, (b) 11-level MMC, (c) 21-level MMC

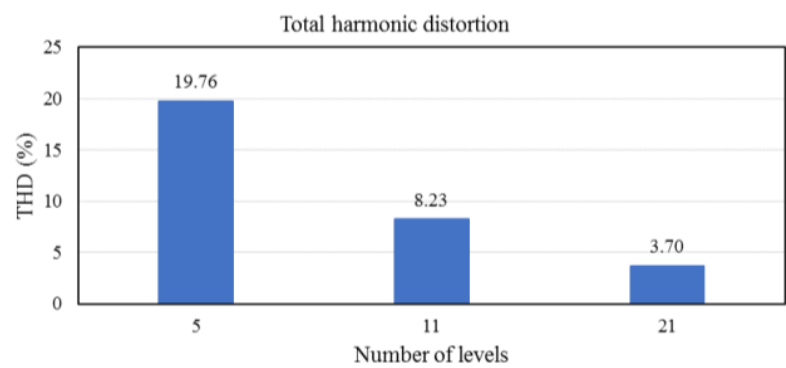

Fig. 5. Total harmonic distortion versus number of levels of MMC

total switching frequencies are the same in three cases, $f_{c t}$ $=3600 \mathrm{~Hz}$. With the higher number of levels of MMC, the sideband harmonic region is wider while the magnitude of harmonics is smaller. This leads a distribution of THD in three cases as shown in Fig. 5. The increasing series of THDs is 21-level MMC (3.7\%), 11-level MMC (8.23\%) and 5-level MMC (19.76\%). In other words, the higher number of levels of MMC can achieve a lower THD. This will be explained as follows. If the level of the MMC is increased, the output voltage will be more nearly sinusoidal waveform. Thus, the harmonics and THD will be decreased.

\section{Conclusion}

This paper has presented an application of DFS algorithm for analyzing harmonics of the MMC. The analytical results used the DFS has been confirmed by comparing with simulated results used the FFT. This means that the DFS can be used to analyze the harmonics in the MMC. Besides, the advantages of the DFS over the FFT is also demonstrated. The DFS does not depend on the simulation time step, and thus it can improve the executive time for calculating harmonics. The MMC with higher number of levels can reduce the THD in the output voltage significantly. In this case, the bandwidth of harmonics is wider, but the magnitude of harmonics is smaller. These conclusions will play an important role in design as well as operation in order to reduce harmonics for the MMC.

\section{Acknowledgement}

This work was supported by the Development of 20MW VSC HVDC for offshore wind-farm interconnection of the Korea Institute of Energy Technology Evaluation and Planning (KETEP), granted financial resource from the Ministry of Trade, Industry \& Energy, Republic of Korea. (No. 2012T100201551).

\section{References}

[1] M. Saeedifard and R. Iravani, "Dynamic Performance of a Modular Multilevel Back-to-Back HVDC System," IEEE Transactions on Power Delivery, vol. 25, no. 4, pp. 2903 2912, Oct. 2010.

[2] Ngoc-Thinh Quach, Ji-Han Ko, Dong-Wan Kim, and EelHwan Kim, "An Application of Proportional-Resonant Controller in MMC-HVDC System under Unbalanced Voltage Conditions," Journal of Electrical Engineering \& Technology, vol. 9, no. 5, pp. 1746-1752, Sept. 2014.

[3] W. R. Bennett, "New Results in the Calculation of Modulation Products," The Bell System Technical Journal, vol. 12, pp. 228-243, Apr. 1933.

[4] D. Grahame Holmes and Thomas A. Lipo, "Pulse Width Modulation for Power Converters - Principles and Practice" Wiley-IEEE Press, 2003.

[5] Gary. W. Chang, Shin-Kuan Chen, Huai-Jhe Su, and PingKuei Wang, "Accurate Assessment of Harmonic and Interharmonic Currents Generated by VSI-Fed Drives under Unbalanced Supply Voltages," IEEE Transactions on Power Delivery, vol. 26, no. 2, pp. 1083-1091, Apr. 2011.

[6] Zhonghui Bing and Jian Sun, "Frequency-Domain Modeling of Multipulse Converters by Double-Fourier Series Method," IEEE Transactions on Power Electronics, vol. 26, no. 12, pp. 3804-3809, Dec. 2011.

[7] C. M. Wu, Wing-Hong Lau, and Henry Shu-Hung Chung, "Analytical Technique for Calculating the Output Harmonics of an H-Bridge Inverter with Dead Time," IEEE Transactions on Circuits and Systems, vol. 46, no. 5, pp. 617-627, May 1999. 